\title{
Differences in manifestations of Marfan syndrome, Ehlers-Danlos syndrome, and Loeys-Dietz syndrome
}

\author{
Josephina A. N. Meester ${ }^{1}$, Aline Verstraeten ${ }^{1}$, Dorien Schepers ${ }^{1}$, Maaike Alaerts ${ }^{1}$, Lut Van Laer ${ }^{1}$, Bart L. \\ Loeys ${ }^{1,2}$ \\ ${ }^{1}$ Center of Medical Genetics, Faculty of Medicine and Health Sciences, University of Antwerp and Antwerp University Hospital, Antwerp, Belgium; \\ ${ }^{2}$ Department of Genetics, Radboud University Medical Center, Nijmegen, The Netherlands \\ Correspondence to: Bart L. Loeys. Center for Medical Genetics, University of Antwerp, Antwerp University Hospital, Prins Boudewijnlaan 43/6, 2650 \\ Antwerp, Belgium. Email: bart.loeys@uantwerpen.be.
}

\begin{abstract}
Many different heritable connective tissue disorders (HCTD) have been described over the past decades. These syndromes often affect the connective tissue of various organ systems, including heart, blood vessels, skin, joints, bone, eyes, and lungs. The discovery of these HCTD was followed by the identification of mutations in a wide range of genes encoding structural proteins, modifying enzymes, or components of the TGF $\beta$-signaling pathway. Three typical examples of HCTD are Marfan syndrome (MFS), Ehlers-Danlos syndrome (EDS), and Loeys-Dietz syndrome (LDS). These syndromes show some degree of phenotypical overlap of cardiovascular, skeletal, and cutaneous features. MFS is typically characterized by cardiovascular, ocular, and skeletal manifestations and is caused by heterozygous mutations in $F B N 1$, coding for the extracellular matrix (ECM) protein fibrillin-1. The most common cardiovascular phenotype involves aortic aneurysm and dissection at the sinuses of Valsalva. LDS is caused by mutations in TGBR1/2, SMAD2/3, or TGFB2/3, all coding for components of the TGF $\beta$-signaling pathway. LDS can be distinguished from MFS by the unique presence of hypertelorism, bifid uvula or cleft palate, and widespread aortic and arterial aneurysm and tortuosity. Compared to MFS, LDS cardiovascular manifestations tend to be more severe. In contrast, no association is reported between LDS and the presence of ectopia lentis, a key distinguishing feature of MFS. Overlapping features between MFS and LDS include scoliosis, pes planus, anterior chest deformity, spontaneous pneumothorax, and dural ectasia. EDS refers to a group of clinically and genetically heterogeneous connective tissue disorders and all subtypes are characterized by variable abnormalities of skin, ligaments and joints, blood vessels, and internal organs. Typical presenting features include joint hypermobility, skin hyperextensibility, and tissue fragility. Up to one quarter of the EDS patients show aortic aneurysmal disease. The latest EDS nosology distinguishes 13 subtypes. Many phenotypic features show overlap between the different subtypes, which makes the clinical diagnosis rather difficult and highlights the importance of molecular diagnostic confirmation.
\end{abstract}

Keywords: Heritable connective tissue disorders (HCTD); Marfan syndrome (MFS); Loeys-Dietz syndrome (LDS); Ehlers-Danlos syndrome (EDS); clinical manifestations

Submitted Jul 01, 2017. Accepted for publication Oct 09, 2017.

doi: 10.21037/acs.2017.11.03

View this article at: http://dx.doi.org/10.21037/acs.2017.11.03

\section{Introduction}

To date, over 200 heritable connective tissue disorders (HCTD) have been described, affecting connective tissue of various organ systems, including heart, blood vessels, bone, eyes, skin, joints, and lungs. The extracellular matrix (ECM) is a highly organized structure, composed of various proteins and polysaccharides. It provides structure and support to the surrounding cells and is involved in differentiation, cell adhesion, and cell-to-cell communication. Furthermore, it is believed that proteins from the ECM also provide 
a regulatory role in sequestering cytokines, for example transforming growth factor-beta (TGF $\beta$ ).

Over the past few decades, many genes have been identified as a cause of HCTD. These genes encode for structural proteins (e.g., FBN1, COL1A1, COL3A1, COL5A1, BGN), modifying enzymes (e.g., ADAMTS2, PLOD1), or components of the TGF $\beta$-signaling pathways (e.g., SMAD2/3, TGFBR1/2 and TGFB2/3) (1). However, these genes do not explain all HCTD patients and many genes still await their discovery.

Marfan syndrome (MFS), Ehlers-Danlos syndrome (EDS), and Loeys-Dietz syndrome (LDS) are examples of HCTD that show some clinical overlap regarding cardiovascular, skeletal, craniofacial, ocular, and cutaneous features. Within this manuscript, unique, and common clinical manifestations of these syndromes are discussed.

\section{Marfan syndrome}

MFS was first described by Antoine Bernard-Jean Marfan in 1896. The prevalence ranges from 1.5 to 17.2 per 100,000 individuals (2) and the phenotype is mainly characterized by cardiovascular, ocular, and skeletal manifestations. The most common cardiovascular phenotype involves aortic aneurysm at the sinuses of Valsalva, which could evolve towards aortic dissection and rupture if undetected and is associated with high mortality rates. However, the first presenting symptom of MFS patients is most commonly due to non-aortic features, including skeletal, ophthalmological, or pulmonary features.

\section{Genetics and pathogenesis}

In 1991, FBN1 mutations were identified as the genetic cause of MFS (3). MFS typically has an autosomal dominant inheritance pattern and is caused by heterozygous mutations in FBN1. However, in very rare cases, homozygous or compound heterozygous mutations have been identified (4). Approximately $95 \%$ of Marfan probands are explained by FBN1 mutations and so far over 1,800 different mutations have been described. In about one third of patients, a de novo mutation is the cause of the disease (5). The previously socalled neonatal form of MFS (nMFS) is typically caused by mutations in the middle part of the FBN1 gene, exons 24-32, comprising transforming growth factor beta binding protein-like domain 3 until calcium-binding epidermal growth factor-like domain 18. Although this neonatal form of MFS was considered as a separate entity in the past, it is now considered as the most severe end of the MFS spectrum, associated childhood mortality $(6,7)$.

$F B N 1$ encodes the fibrillin-1 protein, an important component of the ECM. It serves both a structural and regulatory function. For its structural function, fibrillin- 1 is embedded in the ECM as a component of the microfibrils. Mutations in fibrillin-1 do not only cause weakening of the ECM structure, but will also influence the regulation of the cytokine TGF $\beta$. In aortic tissues of MFS patients, TGF $\beta$ signalling is upregulated and this will result in altered transcription of target genes, including connective tissue growth factor and matrix metalloproteinases.

A more detailed disquisition on the genetic and pathogenic aspects of MFS can be found elsewhere in this issue.

\section{Diagnosis}

In the past, the diagnosis of MFS was made based on the Berlin and Ghent nosology $(8,9)$. Nowadays, the diagnosis is made based on the revised Ghent nosology, established in 2010 (10). This latest nosology puts more emphasis on the presence of cardiovascular features and determines aortic root dilatation and ectopia lentis as the key primary manifestations. In addition, more weight is put onto genetic screening of FBN1. In the absence of any family history, the presence of aortic root dilatation and ectopia lentis is sufficient to make the diagnosis of MFS. However, in the presence of only one of those primary manifestations, the presence of a $F B N 1$ mutation or a systemic score of $\geq 7$ is required to meet the diagnosis of MFS. In the presence of a family history, ectopia lentis, a systemic score of $\geq 7$, or a dilated aorta are all sufficient to make the diagnosis of MFS. The systemic score is based on the presence of several key features, including pectus deformity, wrist and thumb sign, hindfoot deformity, protrusio acetabuli, pneumothorax, dural ectasia, scoliosis, reduced elbow extension, facial features, skin striae, myopia $>3$ diopters, and mitral valve prolapse (MVP) (10).

\section{Cardiovascular features}

The cardiovascular MFS manifestations account for a large proportion of the observed morbidity and mortality. The most prominent cardiovascular feature is a dilation of the aortic root, at the level of the sinus of Valsalva, which is present in the vast majority of patients. However, great variability in rate of dilatation is observed between affected individuals. As a consequence of the dilatation at the aortic root, aortic regurgitation might develop (11). The dilation 
might evolve towards an aneurysm, which in turn can lead to a type A dissection or rupture (12). One possible explanation for the pronounced dilatation at the root of the aorta is related to the high blood pressures it needs to endure. More recent studies also point towards differences in embryological origin of the vascular smooth muscle cells in the root compared to the rest of the aorta (13). A minority of MFS patients (10-20\%) show a dilatation of the descending and abdominal aorta, which can lead to a type $\mathrm{B}$ dissection $(1,12)$. The latter can present as a primary manifestation, but most commonly only occur after the root has undergone surgery. Aortic dilatation or aneurysm can be accompanied by stiffness of the vessel. Stiffness can also be present without a dilatation, suggesting that it could be an early marker of disease (14).

Another important manifestation of MFS is MVP, which is also present in the majority of MFS patients $(15,16)$. Although, only a minority of MFS patients will require surgery as a consequence of MVP. MVP leads to complications like severe mitral valve regurgitation, especially in adolescent women, and can result in heart failure and pulmonary hypertension (12). In addition, calcification of the mitral annulus can already be present at a young age (5). Involvement of other heart valves is also described, including tricuspid valve prolapse and regurgitation and aortic valve insufficiency (12). Although bicuspid aortic valve has been suggested to be more common (17), congenital heart malformations occur far less frequent in MFS when compared to LDS (18).

Dilatation of the main pulmonary artery is also commonly observed in MFS patients, but these rarely dissect or rupture.

In contrast to LDS, in MFS arterial aneurysms are not seen beyond the aorta. Tortuosity at the level of the arteries and aorta, which is very common in LDS, is occasionally observed in MFS and is considered a predictive marker of worse cardiovascular outcome (12).

Dilated cardiomyopathy is an under-recognized clinical feature of the syndrome. Myocardial dysfunction is often considered to be the consequence of valvular insufficiency and ventricular volume overload (12). However, in a subgroup of patients without significant valvular dysfunction, subclinical cardiac dysfunction is described. Indeed, in some patients, dilated cardiomyopathy-like features were observed, that did not correspond to the aneurysmal aortopathy and/or valvulopathy (19). A combination of increased aortic wall stiffness and ECM abnormalities might be underlying these features (19). Additionally, in a minority of patients arrhythmias can be observed in the presence or absence of ventricular dysfunction $(5,12)$.

Neonatal MFS is typically characterized by early onset severe tricuspid and mitral valve regurgitation, which can lead to heart failure, a frequent cause of death in patients with nMFS (7). The valve insufficiencies do occur without significant aortic dilatation.

A higher incidence of pregnancy-related complications, particularly aortic dissection, has been described in patients with MFS (20). However, in LDS the incidence is even higher. The increased risk is related to a combination of hemodynamic changes, hormonal influences, and the change of elastic fiber organization during pregnancy (19).

\section{Skeletal features}

MFS is characterized by many skeletal features which are characterized by a highly variable age of onset. These features are important in the diagnosis, because many patients present with skeletal symptoms. Several skeletal manifestations are a consequence of joint laxity, like pes planus, while others are the result of long bone overgrowth, like dolichostenomelia and arachnodactyly. Joint laxity is observed in the majority of MFS patients, while in LDS it might be dependent on the underlying gene defect. For example, only a minority of LDS patients carrying a SMAD3 mutation suffer from joint laxity (21) but these often present osteoarthritis. Anterior chest deformities (pectus excavatum or carinatum) are the result of a combination of both joint hypermobility and overgrowth of long bones (i.e., the ribs) (5). Most typical are the elongated armspan (more than $5 \%$ difference with stature) and arachnodactyly (evidenced by positive wrist and thumbsigns). The latter tend to be more pronounced in MFS than LDS. Other common skeletal features include vertebral column deformity (scoliosis and thoracolumbar kyphosis), maxillary overbite, reduced elbow extension, protrusion acetabula, hind foot deformity (pes planus) and highly arched palate with crowding of the teeth (12). Lastly, decreased bone density is understudied in MFS, but could be an important cause of the chronic pain experienced by patients with MFS (22). Some reports have suggested a slight increase in fracture rate in MFS patients. Joint contractures are more frequently mentioned in LDS than MFS.

\section{Ocular features}

Ectopia lentis [(sub)luxation of the lenses] is the most typical ocular MFS manifestation and is present in approximately 
$60 \%$ of patients. Surgery is only necessary when normal vision is disturbed. In addition, the globe of the eye is often elongated, resulting in myopia and stretching of the retina, which could in turn lead to retinal detachment (5). Another frequent feature of MFS is corneal flatness, but this is difficult to assess during routine eye exam (23). Cataract and glaucoma also develop more frequently in MFS patients and could be accelerated by the presence of ectopia lentis (5). Lastly, strabismus, although more commonly seen in LDS, is also observed and might cause the development of amblyopia (24).

\section{Pulmonary features}

Considering the pulmonary system, pulmonary blebs are a frequent feature and do evolve towards spontaneous pneumothorax. Obstructive and central sleep apnea is observed more frequently in MFS patients and is related to increased risk for mitral valve surgery, atrial fibrillation, decreased left ventricular ejection fraction, and aortic events $(25,26)$.

\section{Craniofacial features}

MFS is characterized by several craniofacial characteristics, including dolichocephaly, exophthalmos, downslanting palpebral fissures, malar hypoplasia, highly arched palate with tooth crowding, and micro- or retrognathia $(5,12)$. However, these facial features are not specific to distinguish MFS from other connective tissue disorders. In general, the craniofacial features in MFS are less pronounced than those in LDS.

\section{Additional features}

An important cutaneous feature of MFS is the presence of striae distensae, a finding shared with LDS. However, in contrast to LDS and EDS, Marfan patients do typically have a normal texture and elasticity of the skin (5).

Frequent neurological features include dural ectasia, which is also commonly observed in LDS and EDS (5), and spinal arachnoid cysts or diverticula. Severe dural ectasia, including meningoceles, are sometimes accompanied by neurological signs, due to pressure on the nerves. In contrast to LDS, cerebrovascular abnormalities do not occur at an increased frequency in MFS (27).

\section{Loeys-Dietz syndrome}

LDS was first described in 2005 by Bart Loeys and
Harry Dietz. In its most typical presentation, it can be distinguished from MFS by the unique presence of hypertelorism, abnormal uvula (broad, with raphe or bifid) or cleft palate, and widespread aortic and arterial aneurysm and tortuosity. Important additional features that distinguish LDS from MFS include craniosynostosis, clubfoot, joint contractures, and cervical spine instability (12). Compared to MFS, cardiovascular manifestations are more severe and aortic aneurysms tend to dissect or rupture at a smaller diameter and at a younger age. Additionally, aortic aneurysms are not restricted to the root or ascending aorta, but frequently affect aortic side branches and cerebral vessels. Arterial tortuosity mainly effects the neck and head vessels (28). Furthermore, inguinal, umbilical, and hiatal hernia, thin translucent skin, poor wound healing, and atrophic scars are also frequently observed in LDS and not in MFS (12). In contrast, there is no association reported between LDS and the presence of ectopia lentis, a key distinguishing feature of MFS (29). Overlapping features between MFS and LDS include scoliosis, pes planus, anterior chest deformity (pectus excavatum and carinatum), spontaneous pneumothorax, and dural ectasia.

LDS patients most often present with cardiovascular features first, while this is not the case for MFS (11). The remainder of LDS patients often first show skeletal or joint symptoms $(11,30)$.

\section{Genetics}

When LDS was first described, the two different subtypes (type 1 and type 2) were stratified based on the severity of craniofacial and cutaneous features. In 2014, the revised nosology was proposed, stratifying the LDS subtypes (types 1-6) according to the gene that is involved (Table 1) (29). In LDS, components of the TGF $\beta$ signaling pathway are mutated, including the actual cytokines (TGFB2/3), the receptors $(T G F B R 1 / 2)$, and the downstream effectors (SMAD2/3). Paradoxically, these loss-of-function mutations all lead to an increase in TGF- $\beta$ signaling pathway in the aortic wall. Often, de novo inheritance is observed in LDS (31).

\section{Diagnosis}

At present, no formal diagnostic criteria are at hand and a combination of a mutation in one of the known LDS genes and the presence of aortic aneurysms or dissection should be sufficient to make the diagnosis of LDS. Often, family history is also taken into account. Interestingly, intrafamilial 


\begin{tabular}{lll}
\hline \multicolumn{2}{l}{ Table 1 LDS subtypes } & \\
\hline LDS subtype & Gene & Proportion \\
\hline LDS type 1 & TGFBR1 & $20-25 \%$ \\
\hline LDS type 2 & TGFBR2 & $55-60 \%$ \\
\hline LDS type 3 & SMAD3 & $5-10 \%$ \\
\hline LDS type 4 & TGFB2 & $5-10 \%$ \\
\hline LDS type 5 & TGFB3 & $1-5 \%$ \\
\hline LDS type 6 & SMAD2 & $1-5 \%$ \\
\hline LDS, Loeys-Dietz syndrome. & \\
\hline
\end{tabular}

variation and non-penetrance are observed in LDS families and need to be reckoned with (28).

\section{Cardiovascular features}

As in MFS, the main cardiovascular feature in LDS is a dilatation of the aortic root at the level of the sinus of Valsalva and is present in the vast majority of patients (27). Similar to MFS, a great variability in aortic dilatation is observed amongst patients (11). When undetected and untreated, a dilatation could evolve into dissection or rupture, and has been observed as young as 3 months (32). Consequently, these complications are associated with high mortality rates. Similar to MFS, increased aortic stiffness is observed (27). Dilatation of the aortic root can be associated with aortic regurgitation (12). Besides aneurysms affecting the aortic root, LDS is known for its involvement of aneurysms affecting arterial branches of head, neck, lung, lower extremities, and thoracic and abdominal aorta $(21,27,33)$. Dissection of the coronary artery and the internal iliac and superior mesenteric artery have been described without prior dilatation (21,34). Impaired left ventricular systolic function could be the consequence of coronary artery dissection (12). Main pulmonary artery dilatation and aneurysm is frequently seen in severely affected LDS patients (12). Congenital heart malformations are often noted, with bicuspid aortic valve, patent ductus arteriosus and atrial septal defect occurring most often. These congenital heart malformations are only rarely seen in MFS. In LDS patients, pulmonary valve stenosis could also develop over time (21). Similar to MFS, MVP and mitral valve insufficiency are more frequently observed than in the general population $(27,35)$, but appear to be somewhat less common in LDS compared to MFS (36).
With increasing age, left ventricular hypertrophy and atrial fibrillation could develop $(21,27)$, which are both uncommon in MFS. Arterial tortuosity is most prominently affecting the vessels of the head, neck and aorta, but also affects other thoracic and abdominal arteries or can be generalized $(21,27,37,38)$. Cerebrovascular anomalies are frequently diagnosed and include tortuosity, affecting vertebral, internal carotid, cerebral and pericallosal arteries and intracranial aneurysms, typically affecting the vertebral, carotid, basilar, and ophthalmic arteries (21). Cerebral hemorrhage is another major cause of death in LDS patients and is reported as young as 3 years (32). Overall, it can be stated that the aortic and arterial phenotype of patients with mutations in SMAD2, TGFB2 and TGFB3 appears to be less severe than those with mutations in TGFBR1/2, and SMAD3 $(1,12)$. Moreover in TGFB3 families nonpenetrance is commonly observed.

Similar to MFS, a higher incidence of pregnancy-related complications has been described in patients with LDS and include rupture of the gravid uterus and the arteries during or after pregnancy $(20,31)$. Compared to MFS, the incidence of complications might be even higher in LDS $(19,29)$. With regard to surgical outcome, LDS can be distinguished from EDS by the low rate of intraoperative mortality, due to less fragile soft tissues $(28,31,39)$.

\section{Craniofacial features}

Ocular hypertelorism and cleft palate are the two main craniofacial features that characterize LDS. A highly arched palate and bifid uvula, a milder presentation of a cleft palate, are frequently observed (21). Alternatively, the uvula can also have a broad appearance. Both hypertelorism and cleft palate/bifid uvula are characteristic of LDS and not frequently observed in other syndromic aortic aneurysm forms, including MFS. However, recently a new severe syndromic form of thoracic aortic aneurysm was described, caused by mutations in the gene $B G N$, in which uvula anomalies and hypertelorism have also been described (40). In a subset of LDS patients (mostly TGFBR1/2 mutation carries), craniosynostosis is frequently observed, especially in more severely affected patients. All sutures can be involved, but most often the sagittal suture is affected, resulting in dolichocephaly. In addition, the coronal and metopic suture can be involved, resulting in brachycephaly and trigonocephaly, respectively (31). Other LDS craniofacial features are retrognathia, malar flattening, tall and broad forehead, and frontal bossing with a high anterior hair line. 
Patients carrying SMAD3 mutations often display milder craniofacial features compared to other LDS patients (21). LDS patients carrying a mutation in SMAD2 present with downslanting palpebral fissures (12).

\section{Skeletal features}

Considering skeletal features in LDS, a clear overlap with MFS can be appreciated, including pes planus, scoliosis, and pectus deformity. Even though skeletal features due to bone overgrowth can be observed in LDS patients, these are milder compared to MFS. Arachnodactyly is observed in about one third of LDS patients, but true dolichostenomelia, leading to an increase in arm-spanto-height ratio, is far less frequently observed compared to MFS (28). Other key skeletal features in LDS include thoracolumbar kyphosis, joint contractures, asymmetry of the costosternal junction and cervical spine malformation or instability (21). Joint hypermobility is commonly observed and often leads to congenital dislocations of the hip and subluxations of joints. In contrast, reduced joint mobility is characterized by clubfoot and camptodactyly $(29,31)$. Other recurrent skeletal manifestations include spondylolisthesis and acetabular protrusion. In childhood, muscle hypotonia may be present, which might also be observed in several subtypes of EDS. There are also indications that low bone mineral density and osteoporosis with increased risk of fractures and delayed bone healing are involved in the LDS phenotype, but this needs to be investigated on a larger scale (29).

\section{Cutaneous features}

Several cutaneous findings are observed in LDS patients and resemble those observed in EDS. Most typically, velvety, thin and translucent skin with visible veins, easy bruising, poor wound healing and dystrophic scars are observed $(30,31)$. In the absence of craniofacial features, these cutaneous features could be important in distinguishing LDS from MFS (29). Similar to MFS, striae atrophica are often observed (21).

\section{Ocular features}

An important distinguishing feature between LDS and MFS is the lack of ectopia lentis in LDS (28). However, in LDS, other ocular features are observed, including blue sclerae, strabismus, retinal detachment, cataracts, amblyopia, and myopia (29). Interestingly, in comparison with MFS, myopia is less severe in LDS. The presence of blue sclerae seems more specific for LDS, as it is usually not observed in MFS (37). Lastly, retinal tortuosity has been described in LDS, but the clinical significance remains to be determined (29).

\section{Additional features}

Life-threatening complications like spontaneous bowel or spleen rupture and peripartal uterine rupture are described in both EDS and LDS. Spontaneous pneumothorax is common in LDS and MFS. Neuroradiological features include dural ectasia, which is also frequently observed in MFS, and Arnold-Chiari type I malformation, which is not associated with MFS, but is associated with EDS $(12,28,37,41)$. In addition, developmental delay was initially described in LDS patients, whereas this is not associated with MFS, and might be related to the presence of hydrocephalus, craniosynostosis, or intracerebral bleeding $(29,37)$. Dental problems of LDS patients involve defective tooth enamel and dental malocclusion $(12,28)$. Other recurrent findings require further investigation and include, muscle hypoplasia and autoimmune problems, comprising allergic reactions, eczema, food allergies, asthma, and allergic rhinitis (12). Inflammatory bowel disease, including Crohn's disease or ulcerative colitis, and eosinophilic gastrointestinal disease are more common in LDS compared to the general population. Infants and children often suffer from failure to thrive and constipation, which can also be found with other connective tissue disorders $(12,29)$. Patients carrying $S M A D 3$ mutations are typically affected by osteoarthritis at a young age and the vast majority suffers from painful joints $(21,30)$. Other joint anomalies described in $S M A D 3$ patients are intervertebral disc generation, spondylolisthesis, osteochondritis dissecans, and meniscal anomalies. These characteristic joint anomalies are not typically seen in MFS or other subtypes of LDS (21). However, SMAD2 mutation carriers also display a mild degenerative form of osteoarthritis and suffer from painful joints (12,42). Joint pain and fatigue in MFS and LDS seems to be linked to joint hypermobility.

\section{Ehlers-Danlos syndrome}

EDS is a group of connective tissue disorders that is clinically and genetically heterogeneous and all subtypes are characterized by abnormalities of skin, ligaments and joints, blood vessels and internal organs. More specifically, joint hypermobility, skin hyperextensibility and tissue fragility 


\begin{tabular}{llll} 
Table 2 EDS subtypes according to 2017 nosology & \\
EDS subtype & $\begin{array}{l}\text { Inheritance } \\
\text { pattern }\end{array}$ & Gene & Major clinical features \\
\hline $\begin{array}{l}\text { Group A: disorders of collagen primary structure and collagen processing } \\
\text { Classical EDS }\end{array}$ & AD & COL5A/COL5A2 & Skin hyperextensibility, joint hypermobility \\
Vascular EDS & AD & COL3A1 & Thin/translucent skin, characteristic facial appearance, vascular fragility \\
Athrochalasia EDS & AD & COL1A/COL1A2 & $\begin{array}{l}\text { Severe joint hypermobility, congenital hip dislocation, skin } \\
\text { hyperextensibility }\end{array}$ \\
Dermatosparaxis EDS & AR & ADAMTS2 & $\begin{array}{l}\text { Extreme skin fragility, mild joint hypermobility, characteristic facial } \\
\text { features }\end{array}$ \\
Cardiac-valvular EDS & AR & COL1A2 & $\begin{array}{l}\text { Severe cardiac valvular defects, joint hypermobility, skin } \\
\text { hyperextensibility }\end{array}$
\end{tabular}

Group B: disorders of collagen folding and collagen cross-linking
Kyphoscoliotic EDS
AR
PLOD1/FKBP14
Kyphoscoliosis, joint laxity, muscle hypotonia

Group C: disorders of structure and function of myomatrix

$\begin{array}{llll}\text { Classical-like EDS } & \text { AR } & \text { TNXB } & \text { Skin hyperextensibility, joint hypermobility, easy bruising } \\ \text { Myopathic EDS } & \text { AD/AR } & \text { COL12A1 } & \begin{array}{l}\text { Muscle hypotonia/atrophy, proximal joint contractures, distal joint } \\ \text { hypermobility }\end{array}\end{array}$

Group D: disorders of glycosaminoglycan biosynthesis
Spondylodysplastic EDS AR
B4GALT7/B3GALT6
Short stature, muscle hypotonia, bowing of limbs
Musculocontractural AR CHST14/DSE
Congenital contractures, characteristic craniofacial features, skin
EDS fragility/hyperextensibility

Group E: disorders of complement pathway

Periodontal EDS AD C1R/C1S Severe periodontitis, lack of attached gingiva, pretibial plaques

Group F: disorders of intracellular processes

$\begin{array}{llll}\text { Spondylodysplastic EDS } & \text { AR } & \text { SLC39A13 } & \text { Short stature, muscle hypotonia, bowing of limbs } \\ \text { Brittle Cornea syndrome } & \text { AR } & \text { ZNF469/PRDM5 } & \text { Thin cornea, keratoconus, keratoglobus, blue sclerae }\end{array}$

Unresolved forms of EDS

Hypermobile EDS AD ? Joint hypermobility, skin hyperextensibility, smooth velvety skin

$A D$, autosomal dominant; AR, autosomal recessive; EDS, Ehlers-Danlos syndrome; ?, unknown.

are the most typical presenting features. Up to one quarter of the EDS patients show aortic aneurysmal disease (1). The estimated prevalence of EDS ranges from 1:5,000 to 1:25,000 (43). The classic and hypermobile EDS forms account for over $90 \%$ of EDS cases. Vascular EDS (vEDS) represents less than $5 \%$ of cases (44). EDS is caused by mutations in genes coding for collagen fibrils or for proteins involved in the processing of these collagens.

The first international nosology of HCTD was established in 1986 and the different EDS subtypes were numbered (8). However, the Villefranche nosology, established in 1997, recognized six different subtypes and named the subtypes based on the typical clinical characteristics (45). The latest nosology, proposed in 2017, distinguishes 13 subtypes and emphasizes the molecular identification of causative variants (Table 2) (46). Following this nosology, several conditions have been abandoned from the current EDS classification. These encompass the previously called Filamin A related EDS with periventricular nodular heterotopia, in which vascular rupture sporadically 
is observed (1), but also the X-linked EDS with muscle hematoma, the fibronectin-deficient form, and the occipital horn syndrome, a copper deficiency syndrome. The 13 most common, recognized EDS subtypes will be briefly discussed in this manuscript. For each subtype, major and minor criteria are proposed within the 2017 nosology, of which the major criteria involve features that occur in the majority of patients and are rather specific for that subtype, while minor criteria may overlap with other subtypes or HCTDs and might only be present in a small subset of patients (46).

It is worth noting that many clinical findings show overlap between the different EDS subtypes. This makes clinically diagnosing EDS rather difficult and highlights the importance of molecular diagnostic confirmation.

\section{Genetics}

In all subtypes of EDS, except for the hypermobile subtype, mutations in genes encoding fibrillar collagens or genes involved in the modification of collagen are identified. Based on the underlying genetic and pathogenetic mechanism, EDS subtypes are regrouped into disorders of collagen primary structure and collagen processing (group A), collagen folding and collagen cross-linking (group B), structure and function of myomatrix (interface between muscle and ECM) (group C), glycosaminoglycan biosynthesis (group D), complement pathway (group E), and intracellular processes (group F) (Table 2) (46) .

\section{EDS subtypes}

\section{Classical EDS}

Classical EDS is caused by mutations in COLSA1 or COLSA2 and is predominantly characterized by generalized joint hypermobility and skin hyperextensibility with atrophic scarring. In addition, a soft doughy textured skin, easy bruising, skin fragility, mulluscoid pseudotumors, subcutaneous spheroids, hernia, and epicanthal folds can be part of the phenotype, but are less specific for the classical EDS subtype or are present in only a minority of patients (46). Easy bruising during childhood is often the first presenting symptom. Joint hypermobility can be associated with complications like sprains, (sub)luxation, pain, pes planus, and early osteoarthritis. Also muscle hypotonia, scoliosis, pectus deformities, osteopenia, and increased bone fragility are regularly observed and these features can also present in patients with LDS and MFS. In a minority of cases, arterial complications including rupture and dissections are reported and these cases often resemble the vEDS phenotype $(46,47)$. Gastrointestinal symptoms are mostly observed in the hypermobile type of EDS, but are occasionally present in patients with classical EDS, and include dysphagia, reflux, constipation, and diarrhea (47).

\section{Classical-like EDS}

In contrast to classical EDS, which is inherited in an autosomal dominant manner, classical-like EDS is caused by recessive mutations in TNXB (encoding tenascin-X). Like classical EDS, it is characterized by skin hyperextensibility and generalized joint hypermobility (often affecting the small joints of the hands) with or without dislocations (most commonly ankle and shoulder). However, in contrast to classical EDS, no atrophic scarring is observed. Furthermore, easy bruising skin is a major criterion in classical-like EDS. Other recurrent findings include foot deformities, pedal edema (in the absence of cardiac failure), mild proximal and distal muscle weakness, axonal polyneuropathy, atrophy of muscles in hands and feet, acrogeric hands, mallet finger(s), clinodactyly, brachydactyly, and vaginal/uterus/rectal prolapse (46).

\section{Cardiac-valvular EDS}

The cardiac-valvular form of EDS is caused by recessive loss-of-function mutations in COL1A2. Similar to other types of EDS, both skin and joints are affected and form the basis of the major criteria. Skin hyperextensibility, atrophic scars, thin skin and easy bruising are part of the cutaneous phenotype in this EDS subtype. Joint hypermobility can be restricted to small joints, but can also be generalized. Specific for this subtype are the severe progressive cardiacvalvular problems that affect both the aortic and mitral valve (46) and are therefore included in the nosology as a major criterion. Borderline aortic root enlargement has been documented (12). Other recurrent findings include inguinal hernia, pectus deformity, foot deformities with pes planus, and hallux valgus (46).

\section{$v E D S$}

vEDS is inherited in an autosomal dominant manner and is caused by mutations in COL $3 \mathrm{~A} 1$. Its estimated prevalence ranges from $1: 50,000$ to $1: 100,000(12,39)$ and represents approximately $5 \%$ of all EDS cases (44). vEDS is characterized by abnormalities of the skin, joints, hollow organs, and blood vessels and has the worst prognosis amongst all EDS subtypes, due to the rupture of arteries and hollow organs (39). Arterial rupture with 
or without aneurysm at a young age, most often affecting medium-sized abdominal vessels including the renal, iliac, femoral mesenteric, and hepatic arteries, is specific for this subtype $(39,46)$. In several instances, aortic involvement is reported (12). The carotid, subclavian, ulnar, popliteal, and tibial arteries can also be affected (39). Furthermore, other reports mention the presence of abdominal aortic aneurysms and MVP (1). In addition, spontaneous sigmoid colon perforations, and carotid-cavernous sinus fistula formation are major criteria in this subtype $(39,46)$. Uterine ruptures during the third trimester are associated with a significant mortality risk (12). Skin findings in vEDS include easy bruising and bleeding, atrophic scars, and acrogeria. In contrast to other types of EDS that are characterized by hyperextensible, smooth, velvety skin, vEDS is characterized by thin and translucent skin with visible veins, but without hyperextensibility (39). Furthermore, vEDS patients show a characteristic facial appearance, featured by the presence of prominent eyes, thin pitched nose, small lips, hollow cheeks, and lobeless ears (39). Other recurrent findings include spontaneous pneumothorax, clubfoot, congenital hip dislocation, tendon and muscle rupture, recurrent joint subluxation, inguinal hernia, keratoconus, gingival recession and fragility and early onset varicose veins (46). In contrast to classical EDS, large joint hypermobility and skin hyperextensibility are not typical for vEDS.

Surgical interventions are associated with high complication rates, due to the fragility of the soft tissues (39). This is different compared to MFS and LDS, where surgical experience is excellent (31). Furthermore, pregnancy in vEDS patients is considered high risk, due to maternal death as a consequence of uterine rupture or peripartum hemorrhage (39).

Because many features can be very subtle and are also observed in other connective tissue disorders, this makes diagnosing vEDS rather difficult. Complementary diagnosis of pathogenic COL $3 A 1$ mutations help to confirm the diagnosis of vEDS.

\section{Hypermobile EDS}

This subtype of EDS follows an autosomal dominant inheritance pattern with variable clinical expression, but the molecular cause remains unknown to date. Therefore, this group might be a genetically heterogeneous group. The diagnosis of hypermobile EDS should only be assigned in case all criteria are met, in order to reduce heterogeneity. These criteria include the presence of generalized joint hypermobility and absence of unusual skin fragility.
Furthermore, other heritable or acquired connective tissue disorders or alternative diagnoses should be ruled out. Additional features that affect other connective tissues include soft or velvety skin, mild skin hyperextensibility, unexplained striae, bilateral piezogenic papules of the heel, abdominal hernias, atrophic scarring, pelvic floor, uterine, or rectal prolapse, dental crowding and high or narrow palate, arachnodactyly, MVP, aortic root dilatation, and arm span-to-height $\geq 1.05$. Musculoskeletal complications comprise musculoskeletal pain in two or more limbs, chronic widespread pain, and recurrent joint dislocations or joint instability (46). On rare occasions, Chiari malformation type I is reported in these patients, which has also been observed in LDS cases (41). This condition should be distinguished from the joint hypermobility spectrum disorders based on the presence of sufficient of the above listed characteristics (46).

\section{Arthrochalasia EDS}

The arthrochalasia type of EDS is inherited in an autosomal dominant manner and is caused by heterozygous mutations in COL1A1 or COL1A2, affecting the processing of the C-terminal propeptide of COL1A1 or COL1A2. Major findings are congenital bilateral hip dislocation, severe generalized joint hypermobility with dislocations or subluxations, and skin hyperextensibility. Additional minor features include muscle hypotonia, kyphoscoliosis, osteopenia, tissue fragility with atrophic scars, and easy bruising skin (46). Rarely, dissections of medium-sized arteries are described, which, when they do occur, are typically located at the infrarenal region $(1,12)$.

\section{Dermatosparaxis EDS}

Dermatosparaxis EDS is caused by biallelic mutations in the gene ADAMTS2. The phenotype includes extreme skin fragility with skin tears, characteristic craniofacial features, redundant skin, increase palmar wrinkling, severe bruising, umbilical hernia, postnatal growth retardation, short limbs, short hands, short feet, and perinatal complications due to connective tissue fragility, which all serve as major criteria in this disease. Other recurrent features include, soft and doughy skin, skin hyperextensibility, atrophic scars, generalized joint hypermobility, complications due to visceral fragility, delayed motor development, osteopenia, hirsutism, tooth abnormalities, refractive errors, and strabismus (46).

\section{Kyphoscoliotic EDS}

Kyphoscoliotic EDS is caused by recessive mutations in 
PLOD1 or FKBP14. The major criteria of this subtype include congenital muscle hypotonia, kyphoscoliosis, and generalized joint hypermobility with dislocations/ subluxations. Skin findings include skin hyperextensibility and easy bruising skin. Medium sized artery rupture/ aneurysm or aortic dilatation/dissection are rare cardiovascular features that can occur (1). Osteopenia/ osteoporosis, blue sclerae, hernia, pectus deformity, marfanoid habitus, clubfoot, and refractive errors are also frequently observed and form the minor criteria. In comparison, blue sclerae are also observed in LDS. In addition to these general features, gene-specific features can also be assigned. In kyphoscoliotic EDS patients with PLOD1 mutations, skin fragility, scleral and ocular fragility/ rupture, microcornea, and facial dysmorphology are distinguishing features. Congenital hearing impairment, follicular hyperkeratosis, muscle atrophy, and bladder diverticula are specific for kyphoscoliotic patients with FKBP14 mutations (46).

\section{Brittle cornea syndrome}

Brittle cornea syndrome has been added to the EDS spectrum by the 2017 international classification of the EDS, because several patients are clinically suspected to have a form of EDS. This syndrome follows an autosomal recessive inheritance pattern and is caused by mutations in either ZNF469 or PRDM5. Patients mainly display an eye phenotype, including thin cornea, early onset progressive keratoconus, early onset progressive keratoglobus, and blue sclerae. Other less specific ocular features include enucleation or corneal scarring, progressive loss of corneal stromal depth, high myopia, and retinal detachment. Other organ symptoms include deafness, hypercompliant tympanic membranes, developmental dysplasia of the hip, hypotonia in infancy, scoliosis, arachnodactyly, hypermobility of distal joints, pes planus, hallux valgus, mild contractures of fingers, and soft, velvety, and translucent skin (46).

\section{Spondylodysplastic EDS}

Spondylodysplastic EDS is characterized by an autosomal recessive inheritance pattern and is caused by mutations in B4GALT7 or B3GALT6. Specific features for this phenotype are short stature, muscle hypotonia, and bowing of limbs. In addition, skin hyperextensibility, soft and doughy skin, translucent skin, pes planus, delayed motor development, osteopenia, and delayed cognitive development can be part of the phenotype. Depending on the gene that is mutated, specific features can be observed. In B4GALT7 mutation carriers, radioulnar synostosis, bilateral elbow contractures or limited elbow movement, generalized joint hypermobility, single transverse palmar crease, characteristic craniofacial features, characteristic radiographic findings, severe hypermetropia, and clouded cornea are frequently observed. Specific features for B3GALT6 mutation carriers include kyphoscoliosis, joint hypermobility with joint dislocations, joint contractures, peculiar fingers, clubfoot, characteristic craniofacial features, tooth discoloration, and dysplastic teeth (46).

\section{Musculocontractural EDS}

The musculocontractural form of EDS is also inherited in an autosomal recessive manner and caused by mutations in either CHST14 or DSE. It is mainly characterized by congenital multiple contractures, characteristic adduction-flexion contractures, clubfoot, characteristic craniofacial features, increased palmar wrinkling, and characteristic cutaneous features including skin hyperextensibility, easy bruisability, and skin fragility with atrophic scars. Minor criteria involving the skeletal system include recurrent/chronic dislocations, spinal deformities, and peculiar fingers. Other recurrent features include large subcutaneous hematomas, chronic constipation, colonic diverticula, pneumo(hemo)thorax, nephrolithiasis/cystolithiasis, hydronephrosis, cryptorchidism in males, strabismus, refractive errors, and glaucoma/elevated intraocular pressure (46).

\section{Myopathic EDS}

Myopathic EDS can be inherited in both an autosomal dominant or recessive manner and the gene COL12A1 is mutated in this phenotype. Major criteria include congenital muscle hypotonia, muscle atrophy, proximal joint contractures, and hypermobility of distal joints. Other frequently observed features include soft and doughy skin, atrophic scarring, motor developmental delay, and myopathy on muscle biopsy (46).

\section{Periodontal EDS}

This subtype of EDS follows an autosomal dominant inheritance pattern and mutations in $C 1 R$ and $C 1 S$ are the cause of this disease. The phenotype is characterized by severe and early onset intractable periodontitis, lack of attached gingiva, and pretibial plaques. Additional features comprise easy bruising, joint hypermobility (mostly distal joints), skin hyperextensibility and fragility, abnormal scarring, increased rate of infections, hernias, marfanoid facial features, acrogeria, and prominent vasculature (46). 


\section{Rare types of EDS}

Aside from the 13 EDS subtypes recognized by the most recent international classification of EDS [2017], many other rare subtypes of EDS are described in literature (48). However, the discussion of these rare subtypes is beyond the scope of this article.

\section{Conclusions}

MFS, EDS and LDS are examples of HCTD that show some clinical overlap regarding cardiovascular, skeletal, and cutaneous features. Presenting cardiovascular features can lead to complications that are associated with high mortality rates and should therefore be monitored accordingly. Considerable phenotypic overlap is also observed between the different subtypes of LDS and EDS, respectively, which makes the clinical diagnosis difficult and highlights the importance of molecular diagnostic confirmation.

\section{Acknowledgements}

Funding: This research was supported by funding from the University of Antwerp (Lanceringsproject), the Research Foundation Flanders (FWO, Belgium, G.0221.12), The Dutch Heart Foundation (2013T093), the Fondation Leducq (MIBAVA-Leducq 12CVD03). Dr. Loeys is senior clinical investigator of the Research Foundation - Flanders and holds a starting grant from the European Research Council (ERC-StG-2012-30972-BRAVE). JA Meester is a predoctoral researcher of the Research Foundation Flanders. A Verstraeten is a postdoctoral researcher of the Research Foundation - Flanders.

\section{Footnote}

Conflicts of Interest: The authors have no conflicts of interest to declare.

\section{References}

1. Verstraeten A, Alaerts M, Van Laer L, et al. Marfan Syndrome and Related Disorders: 25 Years of Gene Discovery. Hum Mutat 2016;37:524-31.

2. von Kodolitsch Y, De Backer J, Schuler H, et al. Perspectives on the revised Ghent criteria for the diagnosis of Marfan syndrome. Appl Clin Genet 2015;8:137-55 .

3. Dietz HC, Cutting GR, Pyeritz RE, et al. Marfan syndrome caused by a recurrent de novo missense mutation in the fibrillin gene. Nature 1991;352:337-9.

4. Arnaud P, Hanna N, Aubart M, et al. Homozygous and compound heterozygous mutations in the FBN1 gene: unexpected findings in molecular diagnosis of Marfan syndrome. J Med Genet 2017;54:100-3.

5. Judge DP, Dietz HC. Marfan's syndrome. Lancet 2005;366:1965-76.

6. Kirschner R, Hubmacher D, Iyengar G, et al. Classical and neonatal Marfan syndrome mutations in fibrillin-1 cause differential protease susceptibilities and protein function. J Biol Chem 2011;286:32810-23.

7. Maeda J, Kosaki K, Shiono J, et al. Variable severity of cardiovascular phenotypes in patients with an early-onset form of Marfan syndrome harboring FBN1 mutations in exons 24-32. Heart Vessels 2016;31:1717-23.

8. Beighton P, de Paepe A, Danks D, et al. International Nosology of Heritable Disorders of Connective Tissue, Berlin, 1986. Am J Med Genet 1988;29:581-94.

9. De Paepe A, Devereux RB, Dietz HC, et al. Revised diagnostic criteria for the Marfan syndrome. Am J Med Genet 1996;62:417-26.

10. Loeys BL, Dietz HC, Braverman AC, et al. The revised Ghent nosology for the Marfan syndrome. J Med Genet 2010;47:476-85.

11. Attias D, Stheneur C, Roy C, et al. Comparison of clinical presentations and outcomes between patients with TGFBR2 and FBN1 mutations in Marfan syndrome and related disorders. Circulation 2009;120:2541-9.

12. Bradley TJ, Bowdin SC, Morel CF, et al. The Expanding Clinical Spectrum of Extracardiovascular and Cardiovascular Manifestations of Heritable Thoracic Aortic Aneurysm and Dissection. Can J Cardiol 2016;32:86-99.

13. Sherif HM. Heterogeneity in the Segmental Development of the Aortic Tree: Impact on Management of Genetically Triggered Aortic Aneurysms. Aorta (Stamford) 2014;2:186-95.

14. Teixido-Tura G, Redheuil A, Rodriguez-Palomares J, et al. Aortic biomechanics by magnetic resonance: early markers of aortic disease in Marfan syndrome regardless of aortic dilatation? Int J Cardiol 2014;171:56-61.

15. De Backer J, Loeys B, Devos D, et al. A critical analysis of minor cardiovascular criteria in the diagnostic evaluation of patients with Marfan syndrome. Genet Med 2006;8:401-8.

16. Faivre L, Collod-Beroud G, Loeys BL, et al. Effect of mutation type and location on clinical outcome in 1,013 probands with Marfan syndrome or related phenotypes 
and FBN1 mutations: an international study. Am J Hum Genet 2007;81:454-66.

17. Amezcua-Guerra L, Santiago C, Espinola-Zavaleta N, et al. Bicuspid aortic valve: a synergistic factor for aortic dilation and dissection in Marfan syndrome? Rev Invest Clin 2010;62:39-43.

18. Attias D, Mansencal N, Auvert B, et al. Prevalence, characteristics, and outcomes of patients presenting with cardiogenic unilateral pulmonary edema. Circulation 2010;122:1109-15.

19. Takeda N, Yagi H, Hara H, et al. Pathophysiology and Management of Cardiovascular Manifestations in Marfan and Loeys-Dietz Syndromes. Int Heart J 2016;57:271-7.

20. Smith K, Gros B. Pregnancy-related acute aortic dissection in Marfan syndrome: A review of the literature. Congenit Heart Dis 2017;12:251-60.

21. van de Laar IM, van der Linde D, Oei EH, et al. Phenotypic spectrum of the SMAD3-related aneurysmsosteoarthritis syndrome. J Med Genet 2012;49:47-57.

22. Carter N, Duncan E, Wordsworth P. Bone mineral density in adults with Marfan syndrome. Rheumatology (Oxford) 2000;39:307-9.

23. Pyeritz RE, McKusick VA. The Marfan syndrome: diagnosis and management. N Engl J Med 1979;300:772-7.

24. Izquierdo NJ, Traboulsi EI, Enger C, et al. Strabismus in the Marfan syndrome. Am J Ophthalmol 1994;117:632-5.

25. Rybczynski M, Koschyk D, Karmeier A, et al. Frequency of sleep apnea in adults with the Marfan syndrome. Am J Cardiol 2010;105:1836-41.

26. Kohler M, Pitcher A, Blair E, et al. The impact of obstructive sleep apnea on aortic disease in Marfan's syndrome. Respiration 2013;86:39-44.

27. van der Linde D, van de Laar IM, Bertoli-Avella AM, et al. Aggressive cardiovascular phenotype of aneurysmsosteoarthritis syndrome caused by pathogenic SMAD3 variants. J Am Coll Cardiol 2012;60:397-403.

28. Loeys BL, Schwarze U, Holm T, et al. Aneurysm syndromes caused by mutations in the TGF-beta receptor. N Engl J Med 2006;355:788-98.

29. Maccarrick G, Black JH 3rd, Bowdin S, et al. Loeys-Dietz syndrome: a primer for diagnosis and management. Genet Med 2014;16:576-87.

30. van de Laar IM, Oldenburg RA, Pals G, et al. Mutations in SMAD3 cause a syndromic form of aortic aneurysms and dissections with early-onset osteoarthritis. Nat Genet 2011;43:121-6. .

31. Van Hemelrijk C, Renard M, Loeys B. The Loeys-Dietz syndrome: an update for the clinician. Curr Opin Cardiol
2010;25:546-51.

32. Williams JA, Loeys BL, Nwakanma LU, et al. Early surgical experience with Loeys-Dietz: a new syndrome of aggressive thoracic aortic aneurysm disease. Ann Thorac Surg 2007;83:S757-63; discussion S85-90.

33. Martens T, Van Herzeele I, De Ryck F, et al. Multiple aneurysms in a patient with aneurysms-osteoarthritis syndrome. Ann Thorac Surg 2013;95:332-5.

34. Fattori R, Sangiorgio P, Mariucci E, et al. Spontaneous coronary artery dissection in a young woman with LoeysDietz syndrome. Am J Med Genet A 2012;158A:1216-8.

35. Renard M, Callewaert B, Malfait F, et al. Thoracic aorticaneurysm and dissection in association with significant mitral valve disease caused by mutations in TGFB2. Int J Cardiol 2013;165:584-7.

36. Allocco E, Melker M, Rojas-Miguez F, et al. Comparison of Neonatal Abstinence Syndrome Manifestations in Preterm Versus Term Opioid-Exposed Infants. Adv Neonatal Care 2016;16:329-36.

37. Loeys BL, Chen J, Neptune ER, et al. A syndrome of altered cardiovascular, craniofacial, neurocognitive and skeletal development caused by mutations in TGFBR1 or TGFBR2. Nat Genet 2005;37:275-81.

38. Bertoli-Avella AM, Gillis E, Morisaki H, et al. Mutations in a TGF-beta ligand, TGFB3, cause syndromic aortic aneurysms and dissections. J Am Coll Cardiol 2015;65:1324-36.

39. Eagleton MJ. Arterial complications of vascular EhlersDanlos syndrome. J Vasc Surg 2016;64:1869-80.

40. Meester JA, Vandeweyer G, Pintelon I, et al. Loss-offunction mutations in the $\mathrm{X}$-linked biglycan gene cause a severe syndromic form of thoracic aortic aneurysms and dissections. Genet Med 2017;19:386-95.

41. Henderson FC Sr, Austin C, Benzel E, et al. Neurological and spinal manifestations of the Ehlers-Danlos syndromes. Am J Med Genet C Semin Med Genet 2017;175:195-211.

42. Zhang $W$, Zeng Q, Xu Y, et al. Exome sequencing identified a novel SMAD2 mutation in a Chinese family with early onset aortic aneurysms. Clin Chim Acta 2017;468:211-4.

43. Germain DP. Ehlers-Danlos syndrome type IV. Orphanet J Rare Dis 2007;2:32.

44. Papagiannis J. Sudden death due to aortic pathology. Cardiol Young 2017;27:S36-S42.

45. Beighton P, De Paepe A, Steinmann B, et al. EhlersDanlos syndromes: revised nosology, Villefranche, 1997. Ehlers-Danlos National Foundation (USA) and Ehlers-Danlos Support Group (UK). Am J Med Genet 
1998;77:31-7.

46. Malfait F, Francomano C, Byers P, et al. The 2017 international classification of the Ehlers-Danlos syndromes. Am J Med Genet C Semin Med Genet 2017;175:8-26.

47. Bowen JM, Sobey GJ, Burrows NP, et al. Ehlers-Danlos syndrome, classical type. Am J Med Genet C Semin Med Genet 2017;175:27-39.

48. Brady AF, Demirdas S, Fournel-Gigleux S, et al. The Ehlers-Danlos syndromes, rare types. Am J Med Genet C Semin Med Genet 2017;175:70-115.

Cite this article as: Meester JA, Verstraeten A, Schepers D, Alaerts M, Van Laer L, Loeys BL. Differences in manifestations of Marfan syndrome, Ehlers-Danlos syndrome, and LoeysDietz syndrome. Ann Cardiothorac Surg 2017;6(6):582-594. doi: 10.21037/acs.2017.11.03 